Recepción: 01 / 03/ 2018

Aceptación: 15 / 05 / 2018

Publicación: 07 / 07 / 2018
Ciencias de la Salud

Artículo de Investigación

\title{
Embarazo en la adolescencia y su relación con la deserción escolar
}

\author{
Pregnancy in adolescence and its relationship with school dropouts
}

\section{Gravidez na adolescência e sua relação com o abandono escolar}

\section{Gina R. Alonso-Muñiz I \\ gina_am26@hotmail.es}

Solange K. Quijije-Segovia II

solangekarinaquijijesegovia@hotmail.es

Víctor E. Plua-Chancay III

victor.plua@hotmail.com

\author{
Orley L. Tigua-Gutiérrez ${ }^{\text {IV }}$ \\ orleytigua@hotmail.com
}

Alba S. Almendáriz-Parrales V
pasita1607@hotmail.com

Aracely J. Lino-Pionce VI

aracely.lino@hotmail.com

Correspondencia: gina_am26@hotmail.es

I. Magister en Emergencias Médicas, Licenciada en Enfermería, Universidad Estatal del Sur de Manabí, Jipijapa, Ecuador.

II. Magister en Gerencia en Salud para el Desarrollo Local, Licenciada en Enfermería, Universidad Estatal del Sur de Manabí, Jipijapa, Ecuador.

III.

Magister en Gerencia Clínica en Salud Sexual y Reproductiva, Especialista en Cirugía General, Doctor en Medicina y Cirugía, Universidad Estatal del Sur de Manabí, Jipijapa, Ecuador.

IV.

Magister en Emergencias Médicas, Licenciado en Enfermería, Enfermero Responsable de Centro Quirúrgico Hospital General Jipijapa, Docente tutor de internado rotativo de la Universidad Laica Eloy Alfaro de Manabí, Manta, Ecuador

V. Licenciada en Enfermería, Distrito de Salud 13D03 Jipijapa, Puerto López, Ecuador.

VI. Magister en Gerencia y Administración de Salud, Distrito 13D03 Jipijapa, Puerto López, Ecuador. 
Gina R. Alonso-Muñiz; Solange K. Quijije-Segovia; Víctor E. Plua-Chancay; Orley L. Tigua-Gutiérrez; Alba S. Almendáriz-Parrales; Aracely J. Lino-Pionce

\section{Resumen}

En la actualidad el embarazo en adolescentes es un fenómeno de interés mundial debido a la alta incidencia y complicaciones médicas, psicológicas y socioeconómicas, cuyas consecuencias son devastadoras para el entorno familiar, siendo un problema de salud pública y además social que conduce hacia el estrato socioeconómico de la pobreza.

Por ello, el problema que se abordó se centró en el déficit de conocimientos y falta de conciencia que poseen las adolescentes sobre el uso de anticonceptivos y la prevención del embarazo. El método utilizado en esta investigación fue descriptivo en el que se buscó identificar los factores que inducen a tener relaciones sexuales a temprana edad, las técnicas que se manejaron fueron la encuesta, dirigidas a las adolescentes de la "Unidad Educativa Quince de Octubre", y la entrevista a los expertos en este tema.

Dentro de los factores causales del embarazo fue por decisión propia, curiosidad por experimentar una relación sexual, falta de afecto en el hogar y por accidente.

Los resultados determinaron que un porcentaje de adolescentes fueron víctimas de discriminación y mencionaron la falta de apoyo familiar y de la pareja, haciendo que está si piense dejar de estudiar una vez que culmine el embarazo. Por otra parte lo que se evidenció es que hay un alto porcentaje de estudiantes que manifestaron haber sido parte de programas de educación sexual, y otro grupo indico no conocer sobre el tema, razón que hace posible presentar un programa de orientación, prevención y seguimiento sobre la sexualidad dirigida a los y las adolescente de la unidad educativa, donde los protagonistas sean la familia, profesores y la sociedad en sí, porque es aquí donde se produce el problema de desconocimiento y falta de conciencia y por ende donde la intervención familiar juega un rol fundamental para disminuir el embarazo en adolescentes.

Palabras claves: Sexualidad, prevención, métodos anticonceptivos. 


\section{Abstract}

At present, adolescent pregnancy is a phenomenon of worldwide interest due to the high incidence and medical, psychological and socioeconomic complications, whose consequences are devastating for the family environment, being rather a social problem that comes from poverty and continues towards that same address.

Therefore, the problem that was addressed was focused on the lack of knowledge and lack of awareness that adolescents have about the use of contraceptives and the prevention of pregnancy. The method used in this investigation was the descriptive one in which it was sought to identify the factors that induce to have sexual relations at an early age, the techniques that were handled in the survey, directed to the adolescents of the "Educational Unit Fifteen of October", and the interview with the experts on this topic.

Among the causal factors of pregnancy was by choice, by a sexual relationship, lack of affection in the home and by accident.

The results determined that there was no exception in the search for a test and lack of family support due to the state of seriousness of the feelings. It was also evident that there is a higher percentage of students who stated that they had been part of sex education programs, and another indigenous group that did not know about the topic, which is why it can be presented as a final proposal of an orientation, prevention program and monitoring of sexuality. Directed to the children of the educational unit.

Key words: Sexuality, prevention, contraceptive methods. 
Gina R. Alonso-Muñiz; Solange K. Quijije-Segovia; Víctor E. Plua-Chancay; Orley L. Tigua-Gutiérrez; Alba S. Almendáriz-Parrales; Aracely J. Lino-Pionce

\section{Introducción.}

La presente investigación tiene como propósito determinar los factores predeterminantes del embarazo adolescente y su influencia en la deserción escolar en la "Unidad Educativa Quince de Octubre", del Cantón Jipijapa, Provincia de Manabí, Ecuador. Por ello es importante mencionar algunos aspectos que se consideran son relevantes en el desarrollo de la investigación como por ejemplo la concepción sobre adolescencia desde el punto de vista de la salud.

La Organización Mundial de la Salud (OMS) define como adolescencia al “período de la vida en el cual el individuo adquiere la capacidad reproductiva, transita los patrones psicológicos de la niñez a la adultez y consolida la independencia socio-económica (1). Sin embargo, se podría decir que en esta etapa de la vida el ser humano se caracteriza por rápidos cambios físicos que se correlacionan con cambios sociales y psicológicos conducentes a la madurez intelectual y sexual (2), además se carece de capacidad para tomar decisiones acertadas y sensatas.

Según la UNICEF, el período de la vida que va entre los 12 y los 17 años está marcado por profundos cambios en el cuerpo, en la forma de ver el mundo y a los demás. Es una etapa en que el adolescente tiene interrogantes y cuestionamientos, que si no son bien dirigidos tienden a tomar el camino erróneo.

Por otra parte, según la Organización Mundial de la Salud estima que 16 millones de niñas de edades comprendidas entre los 15 y los 19 años dan a luz cada año, y un 95\% de esos nacimientos se producen en países en desarrollo (1). Entonces, Ecuador siendo uno de los países en esta línea, el índice de embarazos en adolescentes es alto, por la pobreza, violencia intrafamiliar, desconocimiento sobre métodos anticonceptivos y sexualidad, y el acceso a la atención integral en salud sexual y reproductiva. 
En todo el mundo, siete países representan por sí solos la mitad de todos los partos en adolescentes: Bangladesh, Brasil, la República Democrática del Congo, Etiopía, la India, Nigeria y los Estados Unidos de América (1).

Los partos en adolescentes como porcentaje de todos los partos oscilan entre alrededor del $2 \%$ en China y el $18 \%$ en América Latina y el Caribe (3). Se corrobora que Ecuador al pertenecer a América Latina tiene un alto índice de embarazos y por ende partos que corresponden a las adolescentes.

Actualmente, el $10 \%$ de los partos corresponde a menores de 19 años y la mortalidad materna es de 2 a 5 veces más alta en las mujeres adolescentes que en las de 20 a 29 años (4). Uno de los factores de riesgo del embarazo adolescente es la muerte materna que está estrechamente relacionada con la edad.

En otro contexto encontrado, en Ecuador aproximadamente 122.301 de las adolescentes son madres, de las cuales el $23 \%$ de ellas tiene entre 10 y 19 años según estadísticas del INEC; esta problemática tiene mayor repercusión en la región Costa con un porcentaje de 55.3\% (5). Es decir un total de 56.128 embarazos entre los años 2005 a 2014, en las edades antes descritas.

Por otro lado el mayor número de nacidos vivos en adolescentes se origina en el rango de madres entre 15 y 19 años. Se evidencia un hallazgo representativo del año 2012 al 2013, período en el cual la tendencia de los embarazos decrece en un $17.83 \%$ en madres adolescentes de 10 a 14 años, y $12.03 \%$ en madres adolescentes de 15 a 19 años (6) 
Gina R. Alonso-Muñiz; Solange K. Quijije-Segovia; Víctor E. Plua-Chancay; Orley L. Tigua-Gutiérrez; Alba S. Almendáriz-Parrales; Aracely J. Lino-Pionce

El embarazo en la adolescencia constituye una de las principales preocupaciones derivadas de la conducta sexual de los jóvenes (7), por la falta de información sobre el uso de anticonceptivos, el no darle valor a su cuerpo y por el inicio de relaciones sexuales a una edad temprana.

La salud general y el futuro económico de padres adolescentes se afectan por las consecuencias de la forzada deserción escolar, la pobreza, la falta de oportunidades y otros aspectos psicosociales (8). Una adolescente embarazada tiende a vivir en un entorno de pobreza por la falta de desarrollo profesional.

El embarazo en la adolescencia tiene gran repercusión en la vida de la mujer, interfiriendo en el desarrollo de sus actividades como estudiante, afectando la salud emocional, el entorno social y económico de la familia. Por otra parte el embarazo en adolescentes es la causa de los muchos hogares o familias disfuncionales, por cuanto a esta edad no se está en capacidad de tomar decisiones convenientes para su bienestar y el de una nueva familia.

Por otro lado Martínez y Félix, afirman también que el embarazo en la adolescencia interrumpe su formación escolar y provoca dificultades para su incorporación al mercado laboral (9). La adolescente bajo esta condición dejara de estudiar por dedicarse al cuidado de su hijo, interviniendo en el desarrollo profesional en el futuro, de tal manera que las posibilidades para acceder a un empleo serán mínimas y no será competitiva en la sociedad, evidentemente el embarazo en esta etapa de la vida será un indicador de pobreza.

El embarazo temprano ocurre en todos los estratos sociales pero predomina en los sectores de menor nivel socioeconómico, y en los países en desarrollo (2). Se considera que la población más vulnerable es la de recursos económicos bajos por la falta de información y el difícil acceso a los 
métodos de anticoncepción. Sin embargo el tema del embarazo es dramático en todos los estratos sociales.

Generalmente el embarazo en esta etapa no es deseado y se constituye en un semillero de eventos adversos, como uniones y matrimonios prematuros de mal pronóstico, rechazo por la familia de origen, desescolarización, hijos que nacen sin una pareja adecuada para su crianza, la inseguridad y desprotección, y mayor exposición al maltrato para el recién nacido (8). Entonces son los cambios en la estructura familiar que han originado una niñez y adolescencia diferentes y podría decirse que es lo que ha hecho que nuestros jóvenes no tengan un modelo ejemplar a seguir.

Un informe de la Comisión Económica para América Latina y el Caribe (CEPAL) reporta que en un conjunto de países latinoamericanos analizados la tasa específica de fecundidad adolescente del quintil más pobre cuadruplica la del quintil más rico (2). Lo que determinan que la población de adolescentes más pobre es la que con mayor frecuencia presenta embarazos.

Dentro de los factores que se asocian a la presentación de embarazo encontramos: familiares, ambientales, sociales, y educacionales, es decir la formación de la joven en relación al empoderamiento de la prevención del embarazo precoz está dada desde su hogar. La deserción escolar de las estudiantes embarazadas en Ecuador ha transcendido a lo largo de los últimos años.

El embarazo trae a menudo graves problemas de tipo social por cuanto, la probabilidad de ser madres en esta etapa aumenta entre las mujeres pertenecientes a los grupos de mayor pobreza y sin protección, aumentando el índice de embarazo de alto riesgo. Los peligros de los que tanto se hablan son variados, desde un sangrado transvaginal, hasta una convulsión, afectando la salud de la madre y también al feto y/o recién nacido, pudiendo ocasionar incluso muerte materna o fetal. 
Gina R. Alonso-Muñiz; Solange K. Quijije-Segovia; Víctor E. Plua-Chancay; Orley L. Tigua-Gutiérrez; Alba S. Almendáriz-Parrales; Aracely J. Lino-Pionce

Uno de los cambios drásticos que produce el embarazo en adolescente es que las chicas ya no tendrán la oportunidad de disfrutar ciertas costumbres que comparten a esta edad, ya no habrá ni vivirán experiencias entre amigos e incluso la relación padres e hijos se verá afectada.

Por otro lado es importante la comunicación en el entorno familiar para fomentar buenas prácticas de sana convivencia y evitar que la adolescente busque información equivocada en lugares y con personas no idóneas.

Durante los últimos años ha existido un gran interés por establecer estrategias dirigidas a prevenir el embarazo "En la actualidad, el embarazo en las adolescentes es un problema de salud pública y trae consigo un número elevado de complicaciones para la salud de la madre y de su hijo." (5).

Se considera a la adolescencia como la etapa en donde se presentan los mayores cambios físicos y psicológicos de las jóvenes, el embarazo conduce alteraciones emocionales en las mujeres, en el ámbito familiar y en las relaciones interpersonales, las nuevas condiciones establecidas ante un embarazo requiere reflexión de la embarazada en el buen cuidado prenatal y hábitos saludables que pueden ayudar a reducir ciertos riesgos, otro factor que también incide en el embarazo a temprana edad además de los ya mencionados es la deserción escolar por cuanto puede haber cierta discriminación por el estado de la adolescente embarazadas ya sea por parte de la familia, condicionando su permanencia en el hogar y poniendo en riesgo su estado de gravidez e incluso dentro de la unidad educativa puede padecer de esta distinción.

A parte de la deserción escolar, el riesgo potencial para las niñas adolescentes de llegar a quedarse embarazada afectaría tanto en el aspecto social, emocional y psicológico, incluyendo además los tempranos contactos sexuales sin el uso adecuado de los anticonceptivos, la presencia de algún 
tipo de enfermedad de transmisión sexual, el uso temprano de alcohol y/u otras drogas, la carencia de un grupo de apoyo o pocos amigos.

La presente investigación pretende beneficiar a las embarazadas y no embarazadas, proponiendo un programa de orientación, prevención y seguimiento en salud sexual y reproductiva incluyendo a las familias y sociedad en general. Trabajando en conjunto y con el apoyo de entidades públicas como: salud y educación de tal manera que se haga eco la información sobre sexualidad logrando disminuir los embarazos, de esta forma el índice de deserción escolar será menor.

\section{Método.}

Para alcanzar los objetivos planteados se desarrolló una investigación de carácter ExploratoriaDescriptiva, donde se utilizaron los métodos de investigación teóricos como el Análisis - Síntesis, Inducción - Deducción y el Hipotético - Deductivo, el método empírico estuvo basado en la observación científica. Se emplearon como instrumento de recolección de datos la encuesta que fue aplicada a las adolescentes de unidad educativa y la entrevista a profesionales especialistas para enriquecer los criterios a propósitos de la investigación. Se aplicaron criterios de inclusión (estudiantes que sean de la institución y estudiantes del sexo femenino entre las edades de 12 a 18 años) y exclusión (estudiantes que no sean de la institución y adolescentes menores de 12 y mayores de 19 años que pertenecen a la entidad educativa), lo que permitió determinar los factores predeterminantes del embarazo adolescente y su influencia en la deserción escolar en la "Unidad Educativa Quince de Octubre”, del Cantón Jipijapa, Provincia de Manabí. Se trabajó con una población total de adolescentes de 386 estudiantes, el nivel de confiabilidad nos da un 93\% cuyos datos fueron procesados en sistema SPSS, y un error admisible de 7\%. La muestra con la que se trabajo fue de 117 estudiantes, el tipo de muestreo optado para la investigación es probabilístico aleatorio simple. La 
Gina R. Alonso-Muñiz; Solange K. Quijije-Segovia; Víctor E. Plua-Chancay; Orley L. Tigua-Gutiérrez; Alba S. Almendáriz-Parrales; Aracely J. Lino-Pionce

misma que se caracteriza porque los elementos de la población tuvieron la probabilidad de ser seleccionados. Los resultados se expresaron en tablas con su respectivo análisis e interpretación.

Al conocer ciertos aspectos relevantes acontecidos en los estudios revisados sobre el embarazo adolescente, se introduce la metodología fenomenológica de dos grandes filósofos clásicos como Edmund Husserl (1859-1938) y su discípulo Martin Heidegger (1889-1976).La fenomenología surge como un excelente método de investigación, muy útil para enfermería, pues permite develar la esencia de los fenómenos asociados al cuidado (10). Por ello, la fenomenología busca comprender al sujeto de cuidado (ser humano), así como, sus experiencias, y vivencias en el entorno en que se desenvuelve.

La fenomenología de Husserl, hace referencia, a que como profesionales de enfermería debemos conocer los aspectos que conllevan a un embarazo adolescente, tomando en cuenta el entorno en que se desenvuelve, los problemas familiares, con la pareja y la sociedad, es decir, se debe descubrir al ser humano, y comprender lo que piensa, conocer sus miedos, temores y sentimientos, y que perspectivas tiene de su vida, de su ser, sin prejuicio de ninguna naturaleza basándonos en el código de ética de enfermeria.

\section{Resultados.}

La encuesta aplicada a las adolescentes embarazadas de la "Unidad Educativa Quince de Octubre" del cantón Jipijapa y las entrevistas a los profesionales expertos en la temática, el $80 \%$ denotan que la causa principal del embarazo en las adolescente se debe a la poca información sobre la sexualidad, lo que se evidencia mediante sus respuestas que carecen de conocimiento acerca del tema, tomando en cuenta que si se han dado programas de capacitación sobre sexualidad, sin embargo es la falta de conciencia en la que se debe hacer énfasis, mediante talleres prácticos, simulacion, no solo a través de conferencias que en ocasiones no genera impacto en ellas, por otro lado el $6 \%$ refieren 
que el desconocimiento se debe a la falta de valores en el hogar, se podría relacionar con la existencia de hogares disfuncionales, en donde la adolescente no tiene un patrón a seguir y un $4 \%$ por otras razones.

Siendo esta etapa en donde mayor atención requiere la adolescente, se debe crear un ambiente de confianza en el entorno familiar, de tal manera que haya comunicación entre padres e hijos en donde se hable con libertad sobre sexualidad sin miedos, ni tabúes.

Otra de las interrogantes fue cuál era la razón que la conllevo a tener relaciones sexuales, el $15 \%$ de las adolescentes indicaron que ha sido por decisión propia, por creer tener la madurez necesaria, porque ellas quisieron, mientras que un $8 \%$ tan solo por curiosidad, y un $1 \%$ indicaron haber tenido relaciones por accidente ( que un $75 \%$ no respondieron.

De las 117 adolescentes encuestadas, el 9\% mencionaron que aun reciben el apoyo de la familia a pesar de su estado de gestación, mientras que el $6 \%$ indico lo contrario, una vez que la familia supo del estado de gravidez de la adolescente, no recibió apoyo moral, ni económico, y el $85 \%$ no se obtuvo respuesta porque no han estado embarazada. La falta de apoyo ha hecho pensar a las adolescentes en querer dejar los estudios una vez que concluya el embarazo, sin embargo esperan terminar el bachillerato.

Por otra parte el $1 \%$ de las adolescentes encuestadas refieren que han sido discriminadas por su condición y el 14\% manifestó que no han sido víctimas de maltrato psicológico, el 85\% no responde porque no están embarazadas. La sociedad estigmatiza a las adolescentes por el embarazo. 
Gina R. Alonso-Muñiz; Solange K. Quijije-Segovia; Víctor E. Plua-Chancay; Orley L. Tigua-Gutiérrez; Alba S. Almendáriz-Parrales; Aracely J. Lino-Pionce

\section{Discusion.}

La comparación de experiencias entre diferentes países demuestra que la prevención efectiva de las conductas de riesgo sexual en adolescentes es el resultado de acciones intersectoriales en las que los medios de comunicación, la educación, la comunidad y la atención sanitaria tienen un papel complementario (11). Con los entes antes mencionados fusionados entre sí, se podrían fortalecer procesos encaminados a disminuir el embarazo adolescente, sería un precedente para que se ejecute en países donde la incidencia de este problema de salud pública es alta.

En estudios realizados en una comunidad en España y otra en Colombia, tuvo como objetivo conocer las percepciones y los imaginarios de las adolescentes frente al embarazo a temprana edad, obteniendo como resultados que las percepciones y creencias sobre un embarazo adolescente están poco definidas y desarrolladas. Las intervenciones en salud sexual y reproductiva dirigidas a la población adolescente deberían tener en cuenta esta diversidad de sentimientos y conductas (12). Esta investigación se inclina hacia los aspectos afectivos y emocionales, socioculturales que se ven afectados por el embarazo.

En Venezuela, se hizo un estudio en una comunidad cuyo objetivo era implementar un programa de intervención educativa que contribuya a modificar los conocimientos de los jóvenes sobre el embarazo en las adolescentes, logrando aumentar el conocimiento sobre el uso de anticonceptivos, complicaciones sobre el embarazo y el aborto, y específicamente modificando conductas (13). Por su parte los investigadores en este estudio revelaron que el nivel de conocimiento no era suficiente por cuanto modificaron y actualizaron temas referentes a sexualidad con la finalidad de disminuir los embarazos. 
En Ecuador, se creó una estrategia para garantizar los derechos sexuales y reproductivos, "Embarazo Adolescente en el Marco de la Estrategia Nacional Intersectorial de Planificación Familiar" (14), denominada ENIPLA, cuyo objetivo es brindar educación, consejería en sexualidad y planificación familiar para la población en general.

Este nace de procesos de largo aliento de la sociedad civil ecuatoriana, que demandan el reconocimiento de derechos sostenidos en la diferencia, es decir, derechos específicos para sectores determinados de la población (14). En vista del problema social y de salud pública el estado ecuatoriano creo este proceso bajo políticas públicas en beneficio de este grupo vulnerable como son las mujeres sexualmente activas y en edad fértil contribuyendo a erradicar la violencia hacia ellas.

Por otro lado en este mismo contexto (ENIPLA) menciona que para poder analizar este fenómeno del embarazo en adolescentes es necesario evaluar los cambios en la sociedad actual especialmente en Ecuador, porque de aquí se origina este problema de salud pública.

Además el Ministerio de Inclusión Económica y Social (MIES), Salud, Educación, Coordinación de Desarrollo Social son parte de esta estrategia con la finalidad de que todos sean parte de este proyecto en el marco de la constitución.

En Ecuador se ha trabajado analizando este fenómeno aplicando procesos en beneficio de esta comunidad tan vulnerable, es así que entidades gubernamentales han creado redes de apoyo, sin embargo no se evidencia en los resultados, porque en vez de disminuir los casos de embarazos en adolescentes, estos van en aumento.

Es relevante este estudio a diferencia de los mencionados, porque además de intervenir con educación en salud sexual y reproductiva, conocer aspectos emocionales y conductuales, lo que 
Gina R. Alonso-Muñiz; Solange K. Quijije-Segovia; Víctor E. Plua-Chancay; Orley L. Tigua-Gutiérrez; Alba S. Almendáriz-Parrales; Aracely J. Lino-Pionce

pretendió presentar fue un programa de participación, orientación sobre sexualidad dirigido no solo a la adolescente sino a la familia y comunidad bajo las directrices del MSP, en trabajo conjunto con el equipo de atención primaria, distrito de educación y MIES. Con un equipo interdisciplinario bien organizado y capacitado para llevar a cabo el desarrollo de este proceso haciendo seguimiento de casos, dando solución y mostrando resultados a la sociedad.

\section{Conclusiones.}

En Ecuador, el 8\% de mujeres de 15-24 años ha tenido relaciones sexuales antes de cumplir los 15 años, y esta cifra se va acumulando al 30\% antes de los 18 años y llega al 46,2\% antes de cumplir 25 años (8).

La causa principal del embarazo de la adolescente se debe a la poca información sobre sexualidad, así como la falta de conciencia de las adolescentes sobre el tema, la secularización de valores, nuevos estilos de vida, las familiar modernas disfuncionales, y falta de reglas en el hogar, entre otras. También se encontraron adolescentes que se embarazaron por otras razones de las cuales no quisieron hablar al respecto.

Entonces cabe recalcar lo importante de la educación sexual desde el entorno familiar, educativo y no tratarlo como un tabú, como algo prohibido, de ahí que surgen las inquietudes en las adolescentes llegando a descubrir por su propia cuenta el sexo pero de manera equivocada. Los datos reflejan que la intervención en educación hasta este momento en Ecuador no alcanza un mayor impacto, tal efecto es evidente en los resultados.

Es relevante recalcar que el pilar fundamental de una adolescente es el seno familiar, de tal manera que es aquí en donde se debería empezar a intervenir en conjunto con quienes forman parte 
del segundo hogar llamado colegio con los tutores, profesores, trabajador social, psicólogos, entre otros.

Los centros de educación como parte de la enseñanza aprendizaje deben diseñar programas participativos, talleres, socio dramas que simulen la maternidad precoz, creando bases de una cultura permanente de orientación en educación sexual integral que cubra todos los espacios e implique a todos los actores del problema de salud.

También la comunidad como sociedad debería velar por el bienestar de sus adolescentes, el Estado mediante el fortalecimiento de normas, deberes, obligaciones, podría establecer trabajos comunitarios para cambiar comportamientos y conductas que conllevan a tener sexo a temprana edad.

En el sector salud, en atención primaria se debería dar prioridad a este grupo vulnerable, haciendo promoción en salud sexual y reproductiva, uso correcto de los métodos anticonceptivos e Infecciones de Trasmisión Sexual (ITS).

El problema principal del embarazo adolescente es la pobreza y falta de oportunidades que irán de generación en generación formando un círculo que difícilmente se romperá, además la morbimortalidad infantil y materna son otras de las complicaciones, todas estas hacen posible que se produzca la deserción escolar.

\section{Bibliografia.}

1. OMS. Embarazo en adolescentes: un problema culturalemnete complejo Washington: World Health Organization; 2009.

2. Federación Latino Americana de Sociedades de Obstetricia y Ginecología. Factores relacionados con el embarazo y la maternidad en menores de 15 años en América Latina y el Caribe. 2010th ed. Orozco LT, editor. Lima: FLASOG; 2011. 
Gina R. Alonso-Muñiz; Solange K. Quijije-Segovia; Víctor E. Plua-Chancay; Orley L. Tigua-Gutiérrez; Alba S. Almendáriz-Parrales; Aracely J. Lino-Pionce

3. OMS/OPS. Boletin Informativo: El embarazo en Adolescentes Washington: World Health Organization; 2015.

4. Castillos L. Desaciertos en la prevencion de embarazos adolescentes. Salud Uninorte. 2016 Octubre; 32(3).

5. Izquierdo K. El embarazo en adolescentes es un problema de salud pública que influye múltiples factores.Acciones de enfermería para la prevención. Examen Complexivo Enfermeria. Machala: Universidad Tecnica de Machala, Unidad Académica de Ciencias Química y de la Salud; 2015.

6. Matute M, Gabriela J. Familia en Cifras- Ecuador 2016. Segunda ed. Loja UTPd, editor. Loja: Ediloja; 2016.

7. Urbina C, Pacheco J. Emabarazos en adolescentes. Revista Peruana de Ginecologia y obstetricia. 2006 junio; 52(2).

8. Gaviria S, Roberto C. Embarazos en adolescentes en America Latina y el Caribe: impacto psicosocial. CCAP. 2013; 12(3).

9. Martinez A, Felix O. Embarazos en adolescentes. Revista de Especialidades Medico-Quirurgicas. 2014 Diciembre; 19(4).

10. Soto C, Vargas I. La Fenomenologia de husserl y Heidegger. Cultura de los cuidados. 2017;: p. 43.

11. Revuelta C. Prevencion del embarazo en la adolescencia. Revista Pediatrica de Atencion Primaria. 2013 Julio-Septiembre; 15(59).

12. Alvarez C, Grande L, Linares M, Cecilia A. Análisis del embarazo adolescente: miradas cualitativas a los casos de Bucaramanga y Jaén. Matronas Profesion. 2017; 18(2).

13. Fleites N, Alvarez Y, Gonzalez I, Diaz J. Intervencion educativa sobre el embarazo en la adolescencia. Medisur. Revista Electronica. 2015 Abril; 13(2).

14. Ministerio de Salud Publica. Embarazo Adolescente en el Marco de la Estrategia Nacional Intersectorial de Planificacion Familiar. 1st ed. Quito: Ministerio de Salud Publica; 2015.

15. Thurnher R. “¡A las cosas mismas!” Acerca de la significación de la máxima fenomenología fundamental en Husserl y Heidegger. Seminarios de filosofia. 1996;: p. 24-44. 


\section{ANEXOS}

TABLAS

Tabla $N^{\circ}$ 1.- Causa principal del embarazo en adolescentes

Causa principal del embarazo

\begin{tabular}{ccc}
\hline VARIABLE & FRECUENCIA & PORCENTAJE \\
\hline Falta de valores & 7 & $6 \%$ \\
Poca información sobre sexualidad & & $80 \%$ \\
& 105 & \\
Otro ( por favor especifique & & $4 \%$ \\
\hline TOTAL & 5 & $\mathbf{1 0 0} \%$ \\
\hline
\end{tabular}

Fuente: Encuesta aplicada a las adolescentes de la Unidad Educativa Quince de Octubre. Elaborado por: los autores

Tabla $N^{\circ}$ 2.- Motivos para iniciar la actividad sexual

Motivo para empezar las relaciones sexuales

VARIABLE

FRECUENCIA

PORCENTAJE

Porque quería tener un hijo

Por curiosidad

9

Por accidente

2

Por presiones grupales

Por necesidad de afecto

Por decisión propia

17

Ninguna Opción

88

TOTAL

117

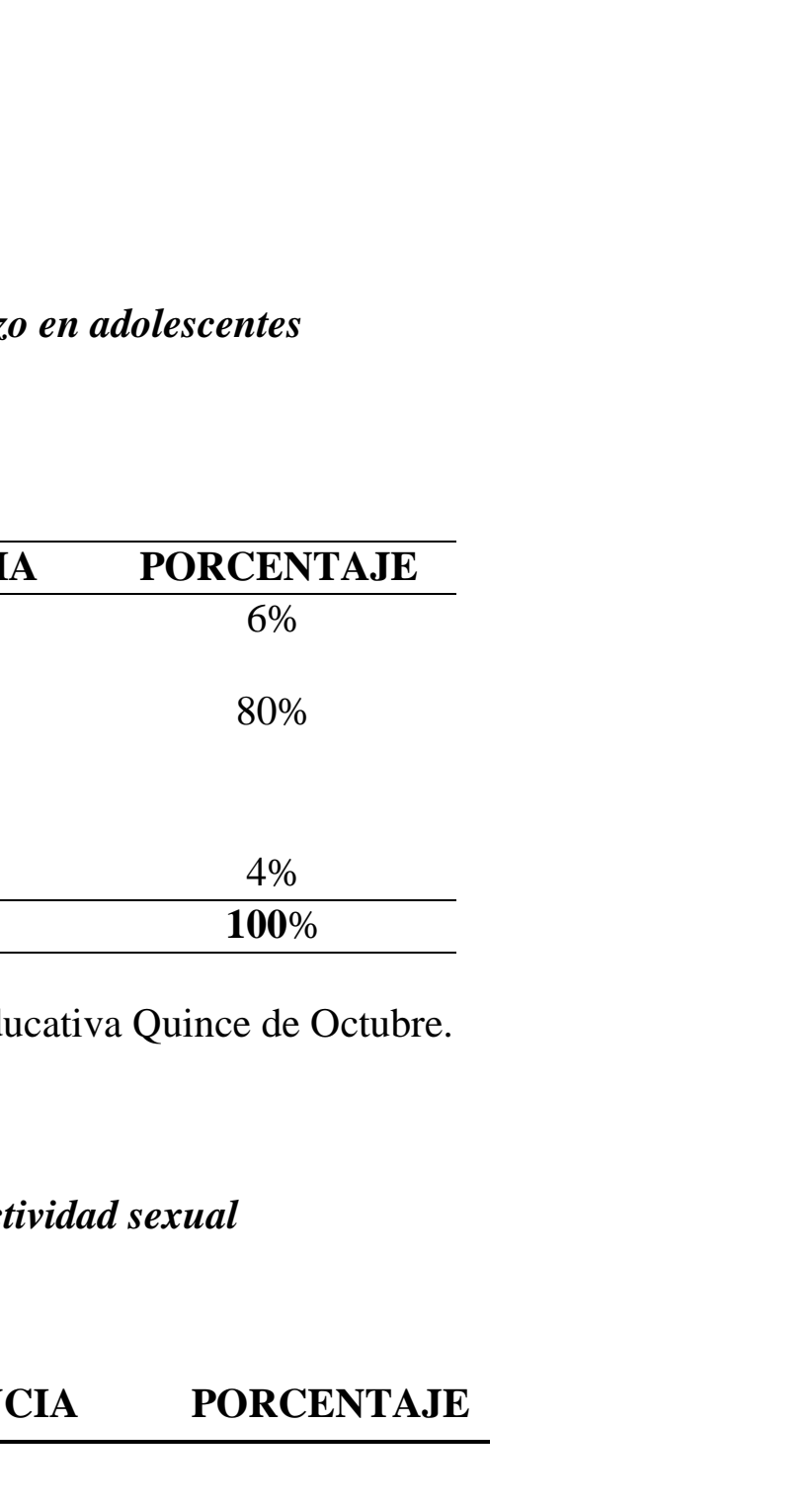

\section{TOTAL}

Fuente: Encuesta aplicada a las adolescentes de la Unidad Educativa Quince de Octubre. Elaborado por: los autores. 
Gina R. Alonso-Muñiz; Solange K. Quijije-Segovia; Víctor E. Plua-Chancay; Orley L. Tigua-Gutiérrez; Alba S. Almendáriz-Parrales; Aracely J. Lino-Pionce

\section{Tabla $N^{\circ}$ 3.- Discriminación por el embarazo}

Discriminación por el embarazo

\begin{tabular}{ccc}
\hline VARIABLE & FRECUENCIA & PORCENTAJE \\
\hline SI & 2 & $1 \%$ \\
NO & 16 & $14 \%$ \\
SIN RESPUESTA & 99 & $85 \%$ \\
\hline TOTAL & $\mathbf{1 1 7}$ & $\mathbf{1 0 0} \%$ \\
\hline
\end{tabular}

Fuente: Encuesta aplicada a las adolescentes de la Unidad Educativa Quince de Octubre.

Elaborado por: los autores.

Tabla $N^{\circ}$ 4.- Apoyo económico y emocional de familia o pareja en la toma de decisiones durante el embarazo

\begin{tabular}{ccc}
\hline VARIABLE & FRECUENCIA & PORCENTAJE \\
\hline SI & 10 & $9 \%$ \\
NO & 8 & $6 \%$ \\
SIN RESPUESTA & 99 & $85 \%$ \\
\hline TOTAL & $\mathbf{1 1 7}$ & $\mathbf{1 0 0} \%$ \\
\hline
\end{tabular}

Fuente: Encuesta aplicada a las adolescentes de la Unidad Educativa Quince de Octubre. Elaborado por: los autores. 